\title{
Experimental research on concrete material components impacting on mechanical properties under uniaxial compression
}

\author{
Jianxin Ding ${ }^{\mathrm{a}}$, Xiaolin Liu ${ }^{\mathrm{b}}$ \\ Changjiang Institute of Survey, Planning, Design and Research, Wuhan 430010, China \\ aemail: dingjx@whu.edu.cn, bemail: liuxiaoling@cjwsjy.com.cn
}

Keywords: building materials; concrete; mortar; aggregate; uniaxial compression

\begin{abstract}
Concrete is one of the important materials in engineering construction. The uniaxial compression experiment reflects its bearing capacity and deformation performance, which is an important method for concrete material properties research. In this paper, the traditional uniaxial compression experiment is used to study the mechanical properties of concrete. Firstly, according to the composition of concrete material, the mortar strength experiment, aggregate content experiment, and aggregate proportion experiment are designed. Then, the standard cube concrete specimens of two-graded are formed according to the experiment design, and the specimens are under standard curing condition in accordance with the requirements of the concrete test specification. The hydraulic servo system is adopted to conduct the uniaxial compression mechanical experiment. The stress-strain curves and damage processes of the mortar specimens as well as concrete specimens under uniaxial compression are obtained. Finally, the experimental results reveal the influence of the water-binder ratio, aggregate content, aggregate proportion on mechanical properties under uniaxial compression, and the reasons are explained and analyzed. The research results have reference value for understanding concrete material properties and benefit to guide concrete structure design.
\end{abstract}

\section{Introduction}

Concrete is one of the important materials in engineering construction, composed of coarse aggregate, fine aggregate, cement hydrates, unhydrated cement particles, bubble and porosity, etc, which is a kind of typical multiphase heterogeneous composite material. The study of its material properties is a hot issue. The concrete material properties affect the design and safety of structures directly, so understanding and mastering its characteristics is an important premise for concrete structure design. At present, as for the conventional concrete material, the mix proportion is usually designed according to the technical requirements, such as the strength, durability, and the deformation index. Firstly, the preliminary water-binder ratio is selected according to the strength requirement. Then selecting the appropriate sand ratio, so the preliminary benchmark mix proportion is determined, and the concrete mechanics experiment is carried out. Finally the laboratory mix proportion is determined by the experiment ${ }^{[1]}$. Therefore, the traditional physical and mechanical experiment is an important approach to study the characteristics of concrete materials, and the results reflect its bearing capacity and deformation performance, at the same time, the damage process and regularity of the concrete specimens are obtained ${ }^{[2]}$.

Usually, concrete can be simply considered as a kind of two phase composite material, which is composited of mortar and aggregate, its macroscopic mechanical properties depends on the properties of the mortar and aggregate and interaction of the two phase components. Many scholars have carried out beneficial exploration on the material properties of concrete through experiment and numerical studies, Liu, etc. carried out the experimental studies, and shown that the compressive strength of a single aggregate graded concrete cubic specimen is obviously low, the aggregate grade has great influence on mechanical properties of concrete ${ }^{[3]}$.Zhang, etc. discussed the factors influencing the strength of concrete, and the mud content of aggregate impacting on concrete strength was studied through experiment ${ }^{[4]}$. Zheng, etc. studied the compressive strength of concrete with different gravel aggregate proportions by experiment, and the destruction theory of interface was used to analyze the result of the experiment ${ }^{[5]}$.Han, etc. studied the impact of the 
coarse aggregate volume fraction on the development of elastic modulus and axial compressive strength of early age concrete, and the prediction model of the development of concrete elastic modulus and axial compressive strength was established based on cement hydration degree ${ }^{[6]}$.Zhu, etc. generated 9 groups of concrete mesoscopic numerical model with different coarse aggregate proportion, and used the finite element analysis system $\mathrm{MFPA}^{2 \mathrm{D}}$ to simulate the damage processes of the numerical specimens under uniaxial compression condition, the law of the numerical simulation is in good agreement with the physical test result ${ }^{[7]}$. The above research results provide guidance to the engineering practice, but due to the discrete and complexities of concrete materials, the research results have certain limitations, at the same time, the whole process of concrete under uniaxial compression, especially the law of the experiment after the peak value is lack of research.

This paper has carried out the experimental analysis of the concrete specimens under uniaxial compression. First of all, on the basis of concrete materials, the experiment is divided into mortar strength experiment, concrete aggregate content experiment, and concrete aggregate proportion experiment, and the mix proportion of the experiments is designed respectively. Then, the standard concrete cube specimens of two-graded are formed in the laboratory. According to the requirements of concrete material mechanics test specification, the specimens are under standard curing condition ${ }^{[8]}$. 28 days later, the uniaxial compression mechanics experiment is carried out on a hydraulic servo machine, and the strain under loading is recorded. The force-displacement curve and the damage processes of the specimens under uniaxial compression are obtained. The results reveal that the effect of the water-binder ratio, aggregate content, aggregate proportion on mechanical properties under uniaxial compression, and the law of the experiment is explained and analyzed.

The aggregate of concrete includes fine aggregate and coarse aggregate, in this paper, the aggregate refers to coarse aggregate, and the fine aggregate is regarded as mortar component.

\section{Experimental design}

Concrete is simplified as a kind of two-phase material, which composed of mortar and aggregate. In order to understand the regularity of physical mechanics and damage processes, the uniaxial compression experiment of concrete specimens is carried out. The experiment is divided into three: (1) The mortar strength experiment. (2) The concrete aggregate content experiment. (3) The concrete aggregate proportion experiment.

\subsection{Mortar strength experiment design}

In order to understand the mechanics law of mortar specimens under different water-binder ratio, the experiment is carried out. Three different water-binder ratios $0.35,0.45$, and 0.55 are used to form three groups standard cube specimens of $150 \mathrm{~mm} \times 150 \mathrm{~mm} \times 150 \mathrm{~mm}$, and each group has three mortar specimens. The mix proportion design is shown in Table.1.

Table.1 The mix proportion design of mortar specimens

\begin{tabular}{ccccc}
\hline Group & $\begin{array}{c}\text { Water-binder } \\
\text { ratio }\end{array}$ & $\begin{array}{c}\text { Water } \\
\left(\mathrm{kg} / \mathrm{m}^{3}\right)\end{array}$ & $\begin{array}{c}\text { Cement } \\
\left(\mathrm{kg} / \mathrm{m}^{3}\right)\end{array}$ & $\begin{array}{c}\text { Sand } \\
\left(\mathrm{kg} / \mathrm{m}^{3}\right)\end{array}$ \\
\hline M1 & 0.35 & 157.5 & & \\
M2 & 0.45 & 202.5 & 450.0 & 1350.0 \\
M3 & 0.55 & 247.5 & & \\
\hline
\end{tabular}

\subsection{Concrete aggregate content experiment design}

In order to understand the effect of aggregate content on the strength of concrete specimens, the experiment is carried out. The experiment is divided into 5 groups, and the aggregate content is $15 \%, 30 \%, 50 \%, 60 \%$, and $70 \%$ respectively. Three standard concrete cube specimens of $150 \mathrm{~mm} \times 150 \mathrm{~mm} \times 150 \mathrm{~mm}$ are formed for each group. The mix proportion design of concrete is shown in Table.2.

Among them, the proportion of $5 \sim 20 \mathrm{~mm}$ small stone and $20 \sim 40 \mathrm{~mm}$ medium stone is 1:1 in the experiment. The gelled material content of C8 group is found to be too low in the experiment, and the specimens can't be formed, so the C8 group is abandoned. 
Table.2 The mix proportion design of aggregate content experiment

\begin{tabular}{cccccccc}
\hline Group & $\begin{array}{c}\text { aggregate } \\
\text { content }\end{array}$ & $\begin{array}{c}\text { Water- } \\
\text { binder } \\
\text { ratio }\end{array}$ & $\begin{array}{c}\text { Water } \\
\left(\mathrm{kg} / \mathrm{m}^{3}\right)\end{array}$ & $\begin{array}{c}\text { Cement } \\
\left(\mathrm{kg} / \mathrm{m}^{3}\right)\end{array}$ & $\begin{array}{c}\text { Sand } \\
\left(\mathrm{kg} / \mathrm{m}^{3}\right)\end{array}$ & $\begin{array}{c}5 \sim 20 \mathrm{~mm} \\
\text { small stone } \\
\left(\mathrm{kg} / \mathrm{m}^{3}\right)\end{array}$ & $\begin{array}{c}20 \sim 40 \mathrm{~mm} \\
\text { medium stone } \\
\left(\mathrm{kg} / \mathrm{m}^{3}\right)\end{array}$ \\
\hline C4 & $15 \%$ & & 206.3 & 458.4 & 1375.3 & 180.0 & 180.0 \\
C5 & $30 \%$ & & 169.9 & 377.5 & 1132.6 & 360.0 & 360.0 \\
C6 & $50 \%$ & 0.45 & 121.3 & 269.7 & 809.0 & 600.0 & 600.0 \\
C7 & $60 \%$ & & 97.1 & 215.7 & 647.2 & 720.0 & 720.0 \\
C8 & $70 \%$ & & 72.8 & 161.8 & 485.4 & 840.0 & 840.0 \\
\hline
\end{tabular}

\subsection{Concrete aggregate proportion experiment design}

In order to understand the effect of concrete aggregate proportion on the specimen strength, the experiment is carried out. The experiment is divided into 7 groups, in each group, supposing the 5 $20 \mathrm{~mm}$ small stone aggregate content is G1, and the $20 \sim 40 \mathrm{~mm}$ medium aggregate stone content is G2.The proportion of $\mathrm{G} 1: \mathrm{G} 2$ is respectively set to be $0.2: 0.8,0.3: 0.7,0.4: 0.6,0.5: 0.5,0.6: 0.4$, 0.7:0.3, 0.8:0.2 in the experiment. In each group, three standard cube concrete specimens of $150 \mathrm{~mm} \times 150 \mathrm{~mm} \times 150 \mathrm{~mm}$ are formed, and the aggregate content is fixed as $40 \%$ in this experiment. The mix proportion design of concrete specimens is shown in Table.3.

Table.3 The mix proportion design of aggregate proportion experiment (aggregate content 40\%)

\begin{tabular}{cccccccc}
\hline Group & $\begin{array}{c}\text { proportion } \\
\text { G1/G2 }\end{array}$ & $\begin{array}{c}\text { Water-bin } \\
\text { der ratio }\end{array}$ & $\begin{array}{c}\text { Water } \\
\left(\mathrm{kg} / \mathrm{m}^{3}\right)\end{array}$ & $\begin{array}{c}\text { Cement } \\
\left(\mathrm{kg} / \mathrm{m}^{3}\right)\end{array}$ & $\begin{array}{c}\text { Sand } \\
\left(\mathrm{kg} / \mathrm{m}^{3}\right)\end{array}$ & $\begin{array}{c}5 \sim 20 \mathrm{~mm} \\
\text { small stone } \\
\left(\mathrm{kg} / \mathrm{m}^{3}\right)\end{array}$ & $\begin{array}{c}20 \sim 40 \mathrm{~mm} \\
\text { medium stone } \\
\left(\mathrm{kg} / \mathrm{m}^{3}\right)\end{array}$ \\
\hline C9 & $0.2 / 0.8$ & & & & & 192.0 & 768.0 \\
C10 & $0.3 / 0.7$ & & & & & 288.0 & 672.0 \\
C11 & $0.4 / 0.6$ & & & & & 384.0 & 576.0 \\
C12 & $0.5 / 0.5$ & 0.45 & \multirow{2}{*}{145.6} & 323.6 & 970.8 & 480.0 & 480.0 \\
C13 & $0.6 / 0.4$ & & & & & 576.0 & 384.0 \\
C14 & $0.7 / 0.3$ & & & & & 672.0 & 288.0 \\
C15 & $0.8 / 0.2$ & & & & & 768.0 & 192.0 \\
\hline
\end{tabular}

\section{The experiment processes and results}

A total of 42 standard cube specimens are formed in the above three experiments, which are shown in Fig.1. After 28 days in standard curing condition, the uniaxial compressive strength experiment of the concrete specimens is carried out in the structure laboratory located in the center of the civil engineering and construction of Wuhan University. The experimental machine is a WAW-1000 microcomputer control electro-hydraulic servo universal testing machine produced by the Changchun keda testing machine manufacturing Co., Ltd, which is shown in Fig.2.The experiment loading is recorded by a personal terminal computer automatically, and the strain is recorded by a XL2118C force \& strain comprehensive parameter tester. In order to avoid the influence of the stress concentration, end loose and uneven, a preloading is adopted for each specimen before the formal loading, which is $30 \%$ of the estimated peak value of axial compression load.

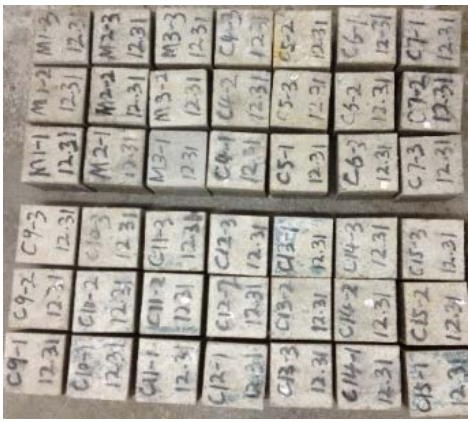

Fig.1 Concrete specimens of each group

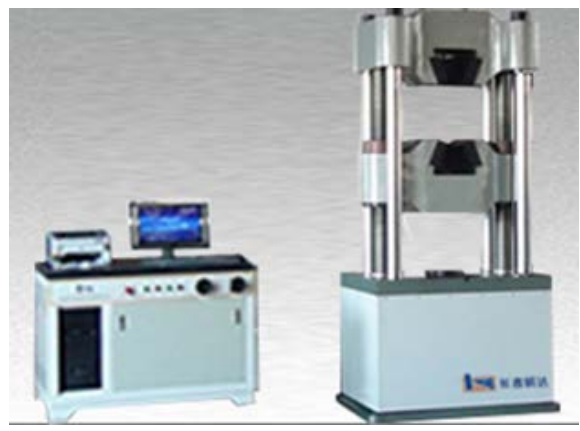

Fig.2 WAW-1000 microcomputer control electro-hydraulic servo universal testing machine

Fig. 3 shows the force-displacement curve of the M2-2 specimen recorded by the servo system. It 
can be seen that:

(1) The compaction stage, after the machine slab falling $8 \mathrm{~mm}$, it arrives at the top of the concrete specimens, and in the initial loading, the mortar specimen is in compaction. The stress-strain curve is a concave type, and the initial nonlinear deformation is formed. The curve slope gradually increases with the loading.

(2) The elastic deformation stage, the stress-strain curve is approximately in a straight line.

(3) The nonlinear stage before the peak, the peak value of M2-2 specimen is $569.1 \mathrm{kN}$.

(4) The strain softening stage, after the peak load, the curve drops rapidly, the mortar specimen no longer bear the load, the specimen is damaged.

(5) The residual stage, the specimen eventually maintains some residual load, and the load is about $120 \mathrm{kN}$.
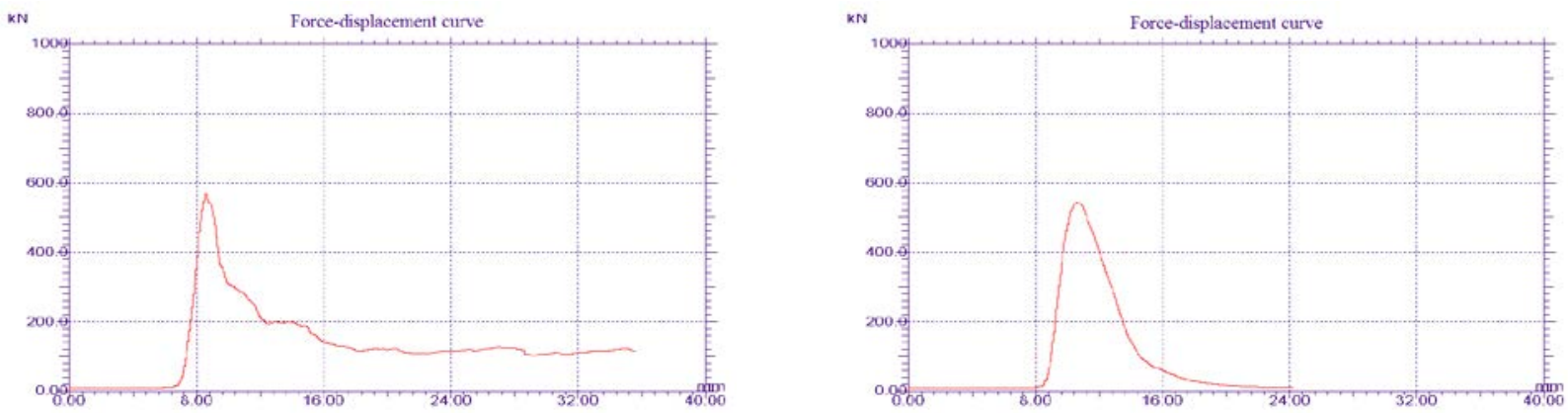

Fig.3 Force-displacement curve of mortar Fig.4 Force-displacement curve of concrete C6-2 M2-2

Fig. 4 shows the force-displacement curve of the C6-2 specimen recorded by the servo system. It can be seen that there are similarities between the force-displacement curve of the concrete specimen and that of the mortar specimen, but there are also differences. The specific characteristics can be seen as follows:

(1) The loading increase and decrease stage of concrete specimens are agree with that of the mortar specimens. There are the initial compaction stage, elastic deformation stage, nonlinear stage, strain softening stage, and the residual stage.

(2) The peak load of the concrete specimens is lower than that of the mortar specimens, and the peak load of concrete C6-2 is about $544.3 \mathrm{kN}$.

(3) The strain softening stage of the concrete specimens after the peak is obviously different with that of the mortar specimens. The strain softening of concrete specimens is smooth, at the same time, the area enclosed by the curve after peak and the axes is significantly greater than that of the mortar specimens, and the brittleness is not obvious as mortar specimens.

(4) The final residual load of concrete specimens is lower than the mortar specimens, and the residual load of concrete C6-2 is about $13 \mathrm{kN}$.

Fig. 5 is the relation curve between compressive strength and water-binder ratio of the mortar groups M1, M2, and M3.It can be seen that the compressive strength of the mortar groups M1, M2, and M3 are about 31MPa, 26MPa, and 21MPa respectively. With the increase of water-binder ratio, the compressive strength of mortar specimens decreases, and the curve declines approximately in a straight line.

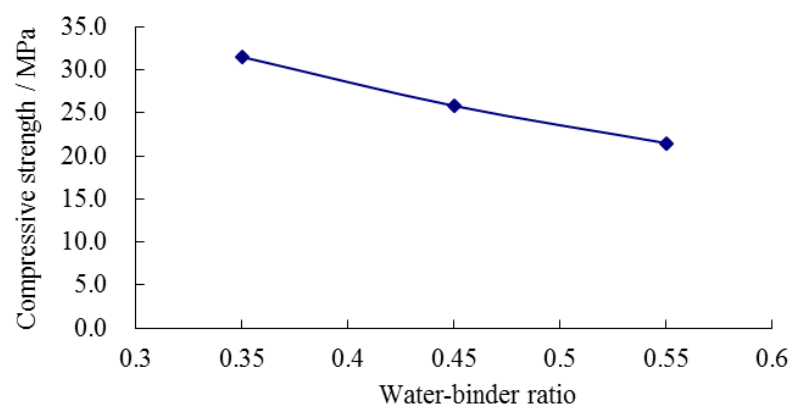

Fig.5 The relation curve between compressive strength and water-binder ratio of mortar specimens 
Fig. 6 shows the compressive strength result of concrete specimens with different aggregate contents, and the result of the aggregate content 0 is for mortar group M2. It can be seen: The compressive strength of the M2 group specimens is about 26MPa. The compressive strength of the C4 group is about $22.5 \mathrm{MPa}$. With the increase of aggregate content, the compressive strength of concrete specimens decreases gradually. The compressive strength of the C5 group is $21.0 \mathrm{MPa}$, and it goes to the lowest point. Then, with the increase of aggregate content, the compressive strength of concrete specimens increases gradually. When the aggregate content is $50 \%$, the highest compressive strength of C6 group is about 23.5MPa. When the aggregate content is more than $50 \%$, the compressive strength gradually reduces, and the compressive strength of concrete specimen C7 group with aggregate content $60 \%$ is about $23.1 \mathrm{MPa}$. The reason of the compressive strength law of the concrete specimens is analyzed here: when the aggregate content is 0 , the compressive strength of mortar is very high. With the increase of aggregate content, the aggregates bear the load alone, and the contact interface area of aggregate and mortar is also gradually increased, which results in the decrease of the compressive strength. After more than $30 \%$ aggregate content, though the aggregate and mortar contact interface area is increased, the aggregate is in close contact with each other and load can be transferred. Therefore, when the aggregate content is about $50 \%$, the compressive strength of concrete specimens is highest. When the aggregate content is more than $50 \%$, the gelled material content is very low in the specimens, which can't supply a good bonding between aggregates, and leads to decrease of the compressive strength. When the aggregate content is more than $60 \%$, the specimen can't be formed.

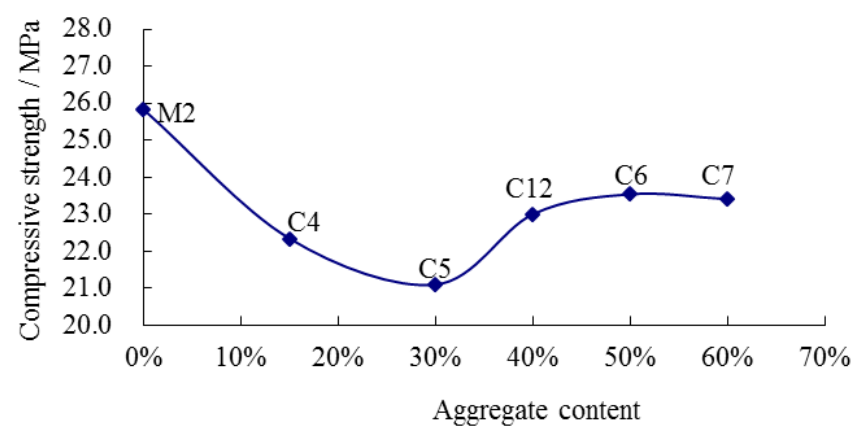

Fig.6 The relation curve between compressive strength and aggregate content of concrete specimens

Fig.7 shows the result of concrete groups C9 C15. It can be seen that the compressive strength of the group C9 specimens is about $22.0 \mathrm{MPa}$. With the increase of small stone aggregate, the compressive strength of concrete specimens increases gradually, and the highest compressive strength of C11 group is about 23.75MPa. Then, with the increase of small stone aggregate, the compressive strength of concrete specimen decreases, and the optimum aggregate proportion of the specimens of the $5 \sim 20 \mathrm{~mm}$ small stone : the $20 \sim 40 \mathrm{~mm}$ medium stone is equal to 0.4:0.6.The result of experiment is agree with many research results (Zheng, etc., 2002; Zhu, etc., 2007).The reason is analyzed here: when the small stone content : medium stone is equal to $0.4: 0.6$, the contact interface area between aggregate and mortar is relatively small, which leads to higher compressive strength.

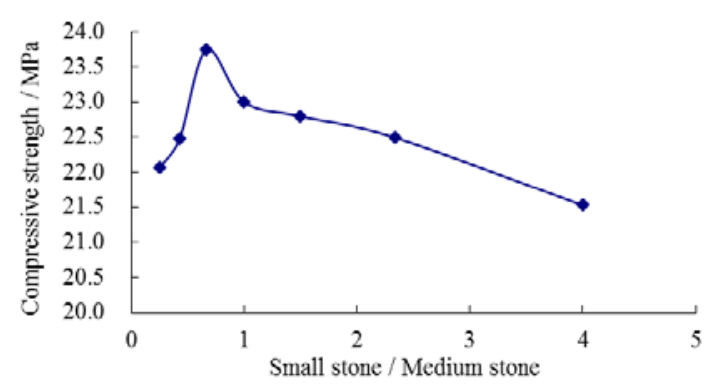

Fig.7 The relation curve between compressive strength and aggregate proportion of concrete specimens 


\section{Conclusion}

This paper has carried out uniaxial compression mechanics experiments of the standard cube concrete specimens of two-graded, and the experiment results reveal the uniaxial compression mechanics of concrete specimens. The main results are as follows:

(1) As for the standard cube mortar specimens, with the increase of water-binder ratio, the compressive strength of mortar specimens decreases.

(2) As for the standard cube concrete specimens of two-graded, with the increase of aggregate content, the compressive strength of concrete specimens gradually reduces to the minimum. Then, with the increase of aggregate content, the compressive strength of concrete specimens increases gradually, when the aggregate content is about $50 \%$, the compressive strength is the highest, when the aggregate content is more than $50 \%$, the compressive strength reduces gradually.

(3) As for the standard cube concrete specimens of two-graded, with the increase of small stone aggregate content, the compressive strength of concrete specimens increases gradually. Then, with the increase of small stone aggregate content, the compressive strength of concrete specimens gradually decreases, and the optimal mix proportion of the small stone: the medium stone is about 0.4:0.6.

Concrete material is a typical heterogeneous composite material, the composition is complex, and many factors affect the material properties. The research achievements in this paper have reference value for understanding the material properties of concrete and benefit to guide concrete engineering design.

\section{References}

[1] Li Yajie, Fang Kunhe. Building materials [M]. Beijing:China WaterPower Press, 2007.

[2] Yang Muqiu, Lin Hong. Experimental research on stress-strain full curves of concrete under uniaxial compression and tension. Shuili xuebao, 1992(6):60-66.

[3] Liu Aiping, Cui Chunlong, Liu Suihai, etc. Study on the relationship between concrete structure and mechanical properties [J]. Geotechnical engineering world, 2005, 8(8):62-64.

[4] Zhang Shangen. Study on influence of the mud content on the strength of concrete [J]. China packaging science and technology review, 2011(26):69-70.

[5] Zheng Jiandong, Xiao Lijun. Relationship between gravel aggregate proportions and water consumptions rate and compressive strength of concrete [J]. Journal of Ningbo university (Natural science \& engineering edition), 2002, 15(2):64-67.

[6] Han Yudong, Zhang Jun, Wang Zhenbo. Effect of volume fraction of coarse aggregate on elastic modulus and compressive strength of concrete [J]. Journal of Harbin Institute of Technology, 2013(4):84-91.

[7] Zhu Fusheng, Xu Shuang, Tang Chunan, etc. Numerical simulation on affection of aggregate gradation to concrete compressive strength [J]. Concrete, 2007(2):8-10.

[8] Ministry of Water Resources of the People's Republic of China. SL352-2006. Test code for hydraulic concrete [S]. Beijing:China WaterPower Press, 2006. 